\title{
Antibiofilm and anti-inflammatory potential of lignans with collegial effect: Secoisolariciresinol diglucoside and sesamin as antimicrobial sources
}

\author{
Ashwini Bhaskar ${ }^{1}$, Shailasree Sekhar ${ }^{2}$, Rajesha Javaraiah ${ }^{1 *}$ \\ ${ }^{1}$ Department of Biochemistry, Yuvaraja's College, University of Mysore, Mysore, India. \\ ${ }^{2}$ Institution of Excellence, Vignana Bhavana, University of Mysore, Mysore, India.
}

\begin{tabular}{l}
\hline ARTICLE INFO \\
\hline Article history: \\
Received on: March 12, 2020 \\
Accepted on: April 23, 2020 \\
Available online: July 30, 2020 \\
\hline
\end{tabular}

\section{Key words:}

SDG, Sesamin, collegial effect, CLSM, bio-film.

\begin{abstract}
Linum usitatissimum (L.), the Flaxseed (FS) and Sesamum indicum (L.), the sesame seeds (SS) are rich sources of lignans, secoisolariciresinol diglucoside (SDG), and Sesamin (Sm), respectively. Synergistic effects of the SDG and Sm lignan samples were investigated in the present study, a first of its kind. The high performance liquid chromatography fingerprint identified the presence of SDG and sesamin in FS and SS. Antimicrobial activity of SDG+Sm combination by disc diffusion in opposition to Bacillus subtilis, Escherichia coli, Klebsiella pneumoniae, Pseudomonas aeruginosa, and Staphylococcus aureus significantly arrests growth of the bacteria in comparison to independent use of SDG and Sm. Bacterial biofilm inhibition capacity of SDG+Sm imaged by confocal laser scanning microscopy revealed the loss of microcolonies. SDG+Sm could inhibit the 15-LOX and COX-2 enzyme at relatively lower concentrations. Furthermore, SDG+Sm quenched free radicals produced by Fenton's reagent studied by DNA-protection assay indicating its robust antioxidant property in protecting the DNA. These results put together encourage the use of a combination of FS and SS seed lignans $\mathrm{SDG}+\mathrm{Sm}$ in a wide range of applications as natural preservatives with pharmacological effects, such as anti-inflammatory agent and aid, in their promotion as nutraceuticals.
\end{abstract}

\section{INTRODUCTION}

Lignans are phytonutrients widely distributed in plants. Linum usitatissimum (L.), the FS, which is the ample origin of iridoid precursors such as secoisolariciresinol diglucoside (SDG) about $>600$ times higher than any plant foods has been reported. A portion of one hundred grams of FS that is powdered could supply a total of 2,230 $\mathrm{kJ}$ or 534 calories in addition to fiber $(28 \mathrm{~g})$, protein $(20 \mathrm{~g})$, and fat $(41 \mathrm{~g})$ per $100 \mathrm{~g}$ of the seed. Sesamum indicum (L.), the sesame seeds (SS), seeds rich in sesamin (Sm) are flavorful with 573 calories per $100 \mathrm{~g}$, protein $(32 \%)$, fat $(49 \%)$, and fiber $(11.8 \mathrm{~g})$. Value-added products for enhancing the value of items of food by the inclusion of an ingredient during processing and/or packaging are found to be attractive. It is received more favorable

*Corresponding Author

Rajesha Javaraiah, Biochemistry Department, Yuvarajas College,

University of Mysore, Mysore, India.E-mail:jrajeshj.51@gmail.com by the consumer. They could be the products, such as breakfast cereals or extruded snacks or ice creams.

Microbial contaminations can make considerable changes in food items and finally affect the quality of these value-added products. Microbial contaminations can adversely affect their shelf life. In contrast, the difference in the intrinsic properties of vegetable oils can change the microbial load of vegetable oils. The fatty acid profile, phenolic compounds, tocopherol, and sterol contents of vegetable oils have a critical role in their intrinsic properties [1]. Benzoic acid, nitrites, and sulfites as antimicrobial agents are in wide use. The antioxidants used include butylated hydroxyanisole, butylated hydroxytoluene, and ascorbic acid. The uses of chemical preservatives are reported to be additive to major health hazardous and toxicity [2]. Thus, the use of natural preservatives has increased and finds acceptance by the consumers as a new approach to overcome these adverse effects $[3,4]$.

Therefore, the reason for this examination was to assess the combinatorial bioactive potencies in lignans from $L$. usitatissimum 

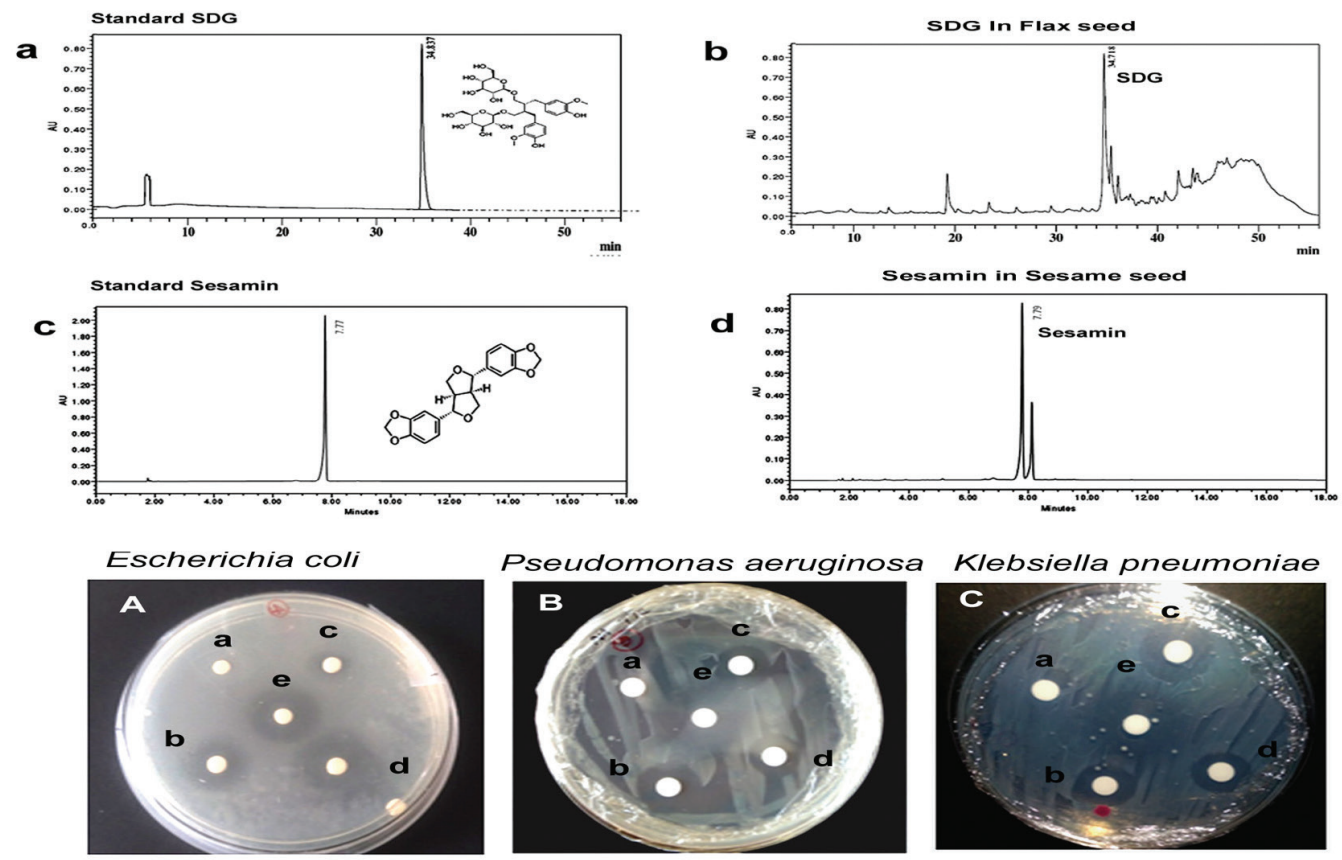

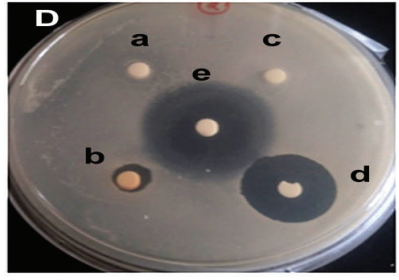

Staphylococcus aureus

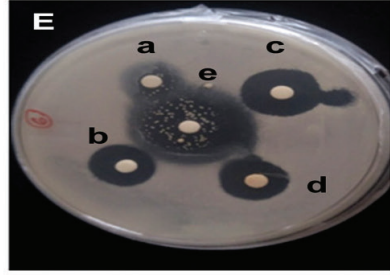

Bacillus subtilis

\section{a - Methanol Control \\ b - SDG \\ c-Sesamin \\ d- SDG+Sm \\ e - Chloramphenicol}

Figure 1. HPLC spectrum of SDG and sesamin from FS and SS respectively. (a) Standard SDG $\left(t_{\mathrm{R}}-34.837\right.$ minutes); (b) presence of SDG in FS ( $t_{\mathrm{R}}-34.718$ minutes); (c) Standard sesamin ( $t_{\mathrm{R}}-7.77$ minutes); (d) presence of sesamin in SS $\left(t_{\mathrm{R}}-7.79\right.$ minutes). $* \mathrm{tR}-$ Retention time and Antibacterial activity of SDG+Sm assayed by disc diffusion method with Gram $(-)$ bacteria E. coli (A); P. aeruginosa (B); K. pneumoniae (C) and gram (+) bacteria S. aureus (D); B. subtilis (E).

(L.), the FS and S. indicum (L.), the SS-SDG and Sm, respectively, a first of its kind. The high performance liquid chromatography (HPLC) fingerprint of $\mathrm{SDG}+\mathrm{Sm}$ was carried out to identify confidently the polyphenols of both the extracts. A differential combination of these was then applied to study the antimicrobial property against Bacillus subtilis, Escherichia coli, Klebsiella pneumoniae, Pseudomonas aeruginosa, Staphylococcus aureus, and outcomes are presented in context to the observations. Other parameters screened were its possible anti-inflammatory activity, evaluated by its efficacy to inhibit human cyclooxygenase-2 (COX2) and 15-lipoxygenase (15-LOX). The DNA protection property was evaluated by DNA-nicking assay using Fenton's reagent.

\section{MATERIALS AND METHODS}

\subsection{Collection and Physicochemical Properties-FS and SS}

Two different varieties of each FS and SS were procured from Haveri and Gulbarga of North Karnataka, India. FS were identified as LCK-5021 and N, N. SS included DS-2 and DS5. The seed samples were preserved for future analysis. In the present study N, N FS and DS-5 SS were used for the studies. Proximate analysis of the seed varieties was carried out according to the Association of Official Analytical Chemists method [5] in triplicates. FS and SS were ground and the contents of ash, moisture, crude fat, crude fiber, total protein, and carbohydrates were analyzed.2.2. HPLC Fingerprinting of SDG and Sm from FS and SSThe lignans SDG+Sm were extracted from the selected variety of FS and SS as described by Rajesh et al. [6], Amarowicz et al. [7], respectively, with slight modifications. The final extract of FS and the standards were subjected to HPLC analysis for fingerprint analysis of its polyphenolic constituents-SDG (Shimadzu, LC-10A, Japan) installed with the RP-C18 column (Shimpack) fitted with a solvent delivery system, photodiode array detectors (SPD 10M AVP) with a particle size of $5 \mu \mathrm{m}$. The mobile phase solvent-A consisted of diluted acetic acid $(1 \% ; \mathrm{v} / \mathrm{v})$, Solvent-B methanol $(100 \%)$ run in direct-slope (1 ml.minute ${ }^{-1}$ ) for 32 minutes and the chromatogram was read at $280 \mathrm{~nm}$. SS was extracted with food-grade hexane for 8 hours with the Soxhlet apparatus. HPLC analysis to detect and confirm the major lignan-Sm with the above specifications is provided. The flow rate was $1 \mathrm{ml} /$ minute for 20 minutes with peak fraction detection at $290 \mathrm{~nm}$. The Sm peak was confirmed in comparison with the standard Sm chromatogram. The retention time for SDG was 34.83 minutes, whereas 7.77 minutes for sesamin (Fig. 1). 


\subsection{Total Phenol Content}

The complete phenol substance of the seed extricates was estimated by the folin-ciocalteau's (FC) technique [8]. Briefly, $2 \% \mathrm{Na}_{2} \mathrm{CO}_{3}$ was mixed with $100 \mu \mathrm{l}$ of the sample and at room temperature, for 2 minutes it was incubated. $100 \mu \mathrm{l}$ of $50 \% \mathrm{FC}$ reagent were added, vortexed thoroughly, and again incubated in the dark for 30 minutes at room temperature. By using a spectrophotometer, the absorbance readings were taken at $765 \mathrm{~nm}$. The unit of phenol contents was expressed as caffeic acid equivalent per gram $(\mathrm{CE} / \mathrm{g})$.

\subsection{Bacterial Strains and Culture Conditions}

Gram (-) bacteria, E. coli (MTCC 724), K. pneumonia (MTCC 661), P. aeruginosa (ATCC 27853) and Gram (+) bacteria, viz., B. subtilis (MTCC 441), and S. aureus (ATCC 25923) were procured from the Institute of Microbial Technology, Chandigarh, India. Preparation of inoculums was done by growing the bacterial cells in broth for 24 hours at $37^{\circ} \mathrm{C}$ in Mueller-Hinton Agar (Himedia, India). These cell suspensions were diluted with sterile Muller-Hinton broth (MHB) to give starting cell checks of about $10^{4} \mathrm{CFU} / \mathrm{ml}$.

Disinfecting lab surfaces before and after completing the antibacterial activity and using proper personal protective equipment (because of the potential splash hazard associated with the manipulation of plant extracts and liquid bacterial cultures, personal protective equipment should include eye protection) was carried out. Contaminated pipette tips and swabs, and all bacterial strains were disposed of by autoclave sterilization after use. Finally, for this activity, laboratory utensils were sterilized (e.g., forceps and bacteria spreader) by dipping in ethanol and flaming with a Bunsen burner.

\subsection{Antibacterial Activity}

\subsubsection{Agar-disc dispersion method}

An aliquot of $50 \mu \mathrm{l}\left(1 \mathrm{mg} \cdot \mathrm{ml}^{-1}\right)$ was presented on the discs $(0.7 \mathrm{~cm}$; Hi-Media) for this disc dispersion experiment. The width of hindrance zones was recorded and was thought about against positive standard-chloramphenicol $(10 \mathrm{ml}$ of $1 \mathrm{mg} / \mathrm{ml})$ and methanol (negative standard; $50 \mu \mathrm{l}$ ) under comparable test conditions.

\subsubsection{Minimum inhibitory concentration}

For measuring minimum inhibitory concentrations (MIC), the method of microdilution with MHB was used [9]. In a 96-well microtiter plate, a two-fold serially diluted samples $(50 \mu \mathrm{l}$ in methanol) of SDG, Sm, and $\mathrm{SDG}+\mathrm{Sm}$ were supplemented by having a concentration extent of $10,5,1.25,0.625,0.3125,0.15625$, 0.078125 , and $0.0390605 \mathrm{mg} / \mathrm{ml}$. To this, around, $50 \mu \mathrm{l}$ of the bacterial suspension with MHB $\left(10^{4} \mathrm{CFU} / \mathrm{ml}\right)$ was supplemented. Control wells were set up with culture medium (sterility control), above said extricate, $50 \mu \mathrm{l}$ of MHB (negative control), methanol (positive control), and $50 \mu \mathrm{l}$ of bacterial suspension in $\mathrm{MHB}$ (positive control). The substance of each well were blended. The MIC was characterized as the most reduced concentration where no viability was seen after 24 hours. This was surveyed dependent on the metabolic movement envisioned as purple shading delivered by the expansion of 2-(4-Iodophenyl)- 3-(4-nitrophenyl)-5- phenyltetrazolium chloride (INT; Sigma-Aldrich St. Louis, MO) broke up in water $(10 \mu \mathrm{l}$ of INT, $2 \mathrm{mg} \cdot \mathrm{ml}-1)$. The plates were brooded in obscurity $\left(37^{\circ} \mathrm{C}\right.$ for 30 minutes) after the expansion of INT. All estimations of MIC values were rehashed in triplicate. Chloramphenicol was utilized as a positive control.

\subsubsection{Confocal laser scanning microscopy study to assay loss of biofilm}

To observe the biofilm structure, confocal laser scanning microscopy (CLSM) LSM 710 (Carl Zeiss, Germany) was used. The samples were prepared according to Zhang et al. [10]. The overnight cultures of $P$. aeruginosa and $S$. aureus were prepared with the absorbance set at $1.5 \pm 2.0(600 \mathrm{~nm})$. The assay was conducted in a 6-well plate. Coverslips were placed in all the wells and above the coverslip; about $2 \mathrm{ml}$ of the media was added. The coverslip with media was maintained as a control. To the test wells, overnight bacterial suspensions $(750 \mu \mathrm{l})$ were introduced. The SDG+Sm extracts $(200$ $\mu 1,750 \mu \mathrm{g})$ were added. The plates were incubated at $37^{\circ} \mathrm{C}$ for 24 hours. The bio-films formed on cover lips in wells were removed carefully. The bio-film was washed with phosphate buffer saline and was stained with ethidium bromide $(20 \mu \mathrm{l} ; 1 \mathrm{mg}$ in $400 \mu \mathrm{l}$; HiMedia, India). After three washes, the coverslips with stained biofilm were placed on a glass slide in an inverted position. CLSM images of the samples were imaged and results were presented.

\subsection{Anti-Inflammatory Activity}

\subsubsection{Lipoxygenase (15-LOX) inhibition}

A spectrophotometric test for assurance of soybean 15-LOX (5 $\mu \mathrm{g}$; Sigma- Aldrich, St. Louis, MO) activity was done with 0.2 $\mu \mathrm{M}$ linoleic acid (substrate; Sigma-Aldrich, St. Louis, MO) with buffer [0.2 M borate buffer ( $\mathrm{pH} 9.0$ )]. For the inhibition studies, the SDG, Sm, and SDG+Sm at 10, 25, 50, 75, and $100 \mu \mathrm{g}$ per $\mathrm{ml}$ were used. The hydroperoxide content values were estimated. The activity of LOX was computed as reported earlier [11].

\subsubsection{Human cyclooxygenase (COX)-2 inhibition}

Human cyclooxygenase (COX)-2 reticence kit was purchased from Cayman Chemicals, Ann Arbor, MI. Computation of COX-2 inhibition by $\mathrm{SDG}, \mathrm{Sm}$, and $\mathrm{SDG}+\mathrm{Sm}$ was done by using a COX2 inhibitor (human) screening kit (Cayman, Ann Arbor, MI) by colorimetric estimation. The $100 \mu \mathrm{g}$ of the samples of SDG, Sm, and $\mathrm{SDG}+\mathrm{Sm}$ were tested for inhibition reticence studies as per the producer's procedure. In a microtitre plate reader (Varioskan Flash with SkanIt) with Software RE 2.4.3, the readings were recorded at $415 \mathrm{~nm}$.

\subsection{DNA Protection Assay}

By using supercoiled plasmid pBR322 (Merck Biosciences, Bangalore, India), DNA-protection protocol was performed [12]. The plasmid DNA with Fenton reagent subjected for 30 minutes reaction served as a control under akin conditions. DNA defense mechanism against Fenton's reagent was estimated by incubating $10 \mu \mathrm{g}$ of the samples (SDG, Sm, and SDG+Sm) with plasmid DNA $(0.5 \mu \mathrm{g})$ for 30 minutes at $37^{\circ} \mathrm{C}$ and further to Fenton reagent for 30 minutes. The samples were separated using a $1 \%$ agarose gel. 
Table 1. Chemical composition of L. usitatissimum (L.), FS and S. indicum (L.), SS.

\begin{tabular}{clcc} 
Sl. No. & \multicolumn{1}{c}{ Parameters (\%) } & \multicolumn{2}{c}{ Percent composition $^{\mathrm{a}}$} \\
& & FS (N,N) & SS (DS-5) \\
1 & Moisture & 4.8 & 4.4 \\
2 & Total ash & 4.0 & 4.0 \\
3 & Total fiber & 22.0 & 6.0 \\
4 & Oil & 40.4 & 59.3 \\
5 & Protein & 21.3 & 19.0 \\
6 & Carbohydrate & 7.5 & 7.3 \\
\hline
\end{tabular}

${ }^{\mathrm{a}} \mathrm{All}$ the values were means of three mean $\pm \mathrm{SD}(n=3)$ measurements.

The results were documented using the $\mathrm{XR}+$ Molecular Imager Gel documentation system (Bio-Rad, USA).

\subsection{Statistical Investigation}

All analyses and estimations were made in triplicate. The variables are communicated as the mean \pm S.E.M. The outcomes were exposed to variance investigation followed by the Turkeys test to break down contrasts among $\mathrm{SDG}+\mathrm{Sm}$ and control conditions. Factually critical contrasts $(p<0.05 / 0.01)$ were appeared.

\section{RESULTS}

\subsection{Physicochemical Properties and Total Phenol Content}

Preliminary phytochemical investigation of the selected FS and SS cultivars uncovered the nearness of intriguing mixes. Table 1 introduces the proximate structure of FS and SS blend. The significant low moisture esteems would permit putting away seeds for quite a while since higher dampness substance could cause deterioration of unsaturated fats by microbial activity.

The total phenol content in the extracts of FS (N, N) and SS (DS5) was $1.84 \pm 0.02 \mathrm{mg}$ and $0.86 \pm 0.03 \mathrm{mg}$ (Gallic acid equivalent per gram dry weight), respectively.

\subsection{HPLC Fingerprint of SDG and Sm from FS and SS}

The SDG and Sm iridoid contents from the FS and SS were identified and evaluated by HPLC. The reference standards of SDG and sesamin $\left(10 \mu \mathrm{l}\right.$ of $\left.1 \mathrm{mg} \cdot \mathrm{ml}^{-1}\right)$ were used (Fig. 1). The HPLC analysis revealed the presence of SDG and sesamin. The standard SDG eluted at 34.83 minutes (Fig. 1a) and sesamin eluted at 7.8 minutes (Fig. 1c). The presence of these two iridoids in FS (SDG; Fig. 1b) and SS extracts (sesamin; Fig. 1d) were identified in comparison to the reference standards. The amount of total SDG and sesamin contents in purified extracts were calculated in comparison to standards. SDG and sesamin were estimated to be 23.9 and 4.7 g. $\mathrm{kg}^{-1}$ in seeds of N, N and DS-05 varieties, respectively.

\subsection{Antibacterial Activity}

\subsubsection{Agar-disc diffusion method}

The zone of inhibition studies by the agar-disc diffusion method exhibited a clear antimicrobial effect of the combined use of SDG and $\mathrm{Sm}$ as visualized and presented (Fig. 1A-E). Statistically
Table 2. Minimum inhibitory concentration $\left(\mu \mathrm{g} \cdot \mathrm{ml}^{-1}\right)$ of SDG and sesamin.

\begin{tabular}{lccccc}
\multicolumn{1}{c}{ Samples } & $\begin{array}{c}\text { E. coli } \\
(-\mathrm{ve})\end{array}$ & $\begin{array}{c}\text { P. aeruginosa } \\
(-\mathrm{ve})\end{array}$ & $\begin{array}{c}\text { K. pneumoniae } \\
(-\mathbf{v e})\end{array}$ & $\begin{array}{c}\text { S. aureus } \\
(+\mathrm{ve})\end{array}$ & $\begin{array}{c}\text { B. subtilis } \\
(+\mathrm{ve})\end{array}$ \\
SDG & $132.2^{\mathrm{b}}$ & $136.4^{\mathrm{b}}$ & $156.4^{\mathrm{e}}$ & $146.8^{\mathrm{cd}}$ & $138.7^{\mathrm{b}}$ \\
Sm & $135.3^{\mathrm{b}}$ & $143.6^{\mathrm{c}}$ & $141.2^{\mathrm{c}}$ & $148.9^{\mathrm{cd}}$ & $136.4^{\mathrm{b}}$ \\
SDG+Sm & $102.5^{\mathrm{a}}$ & $104.1^{\mathrm{a}}$ & $103.6^{\mathrm{a}}$ & $127.3^{\mathrm{a}}$ & $110.1^{\mathrm{a}}$ \\
Chloramphenicol & 22.9 & $31.4^{4}$ & 15.3 & 62.1 & 31.4 \\
\hline
\end{tabular}

All the values were means of three mean $\pm \mathrm{SD}(n=3)$ measurements.

significant inhibition of both Gram $(-)$ and Gram $(+)$ bacteria was recorded. The SDG+Sm were found to exert a high and maximum significant antibacterial effect against E. coli, S. aureus, and B. subtilis (Fig. 1A, D, and E).

\subsubsection{Minimum inhibitory concentration}

The screening of the samples was done by MIC investigation for their bacterial efficacy by following the micro-dilution (broth) strategy. As an observation, $\mathrm{SDG}+\mathrm{Sm}$ was seen very effective against all the tested bacterial strains tested (Table 2 and Fig. 2f). It could inhibit most of the $E$. coli growth with the best MIC of $102.5 \mu \mathrm{g} . \mathrm{ml}-1$ in comparison when SDG and Sm were used independently.

\subsection{CLSM Observation of Biofilm}

Gram-positive strain, S. aureus (ATCC 25923) and Gram-negative strain, $P$. aeruginosa (ATCC 27853) commonly reported as biofilm-forming strains were included in this study. From sample observations of controls, $S$. aureus (Fig. 2a) and $P$. aeruginosa (Fig. 2c) formed an uninterrupted biofilm on the support as was expected. The addition of SDG+Sm extracts $(200 \mu \mathrm{l}, 750 \mu \mathrm{g}) \mathrm{a}$ loss in the biofilm was observed. A significant biofilm inhibition was visualized by CLSM studies as a breakdown of the biofilm architecture and with decreased micro-colonies on the cover-slips containing $S$. aureus (Fig. 2b) and P. aeruginosa (Fig. 2d).

\subsection{Anti-Inflammatory Activity}

\subsubsection{Lipoxygenase (15-LOX) inhibition}

The SDG, Sm, and their combination, SDG+Sm could inhibit 15LOX to various capacities (Table 3 ). The combination displayed powerful restraining $94.37 \% \mathrm{LOX}$ action with $\mathrm{IC}_{50}$ of $35 \pm 0.53$ which was strained by SDG-68.26\%, Sm-67.07\% prohibition, respectively.

\subsubsection{Human cyclooxygenase (COX)-2 inhibition}

Inhibitory effects of the SDG, Sm, and their combination, by in vitro enzymatic exercises $\mathrm{SDG}+\mathrm{Sm}$ at $100 \mu \mathrm{g}$ estimated against human COX-2 demonstrated SDG + Sm inhibited human COX-2 by $73.56 \pm 2.02 \%$. SDG alone inhibited it by $62.18 \pm 3.14 \%$ and Sm could inhibit it alone by $56 \pm 2.11 \%$ of the activity (Table 3 ).

\subsection{DNA Protection Assay}

To research the adequacy of the SDG, Sm, and their combination, SDG + Sm shielding DNA from harm, plasmid DNA (pBR322) 


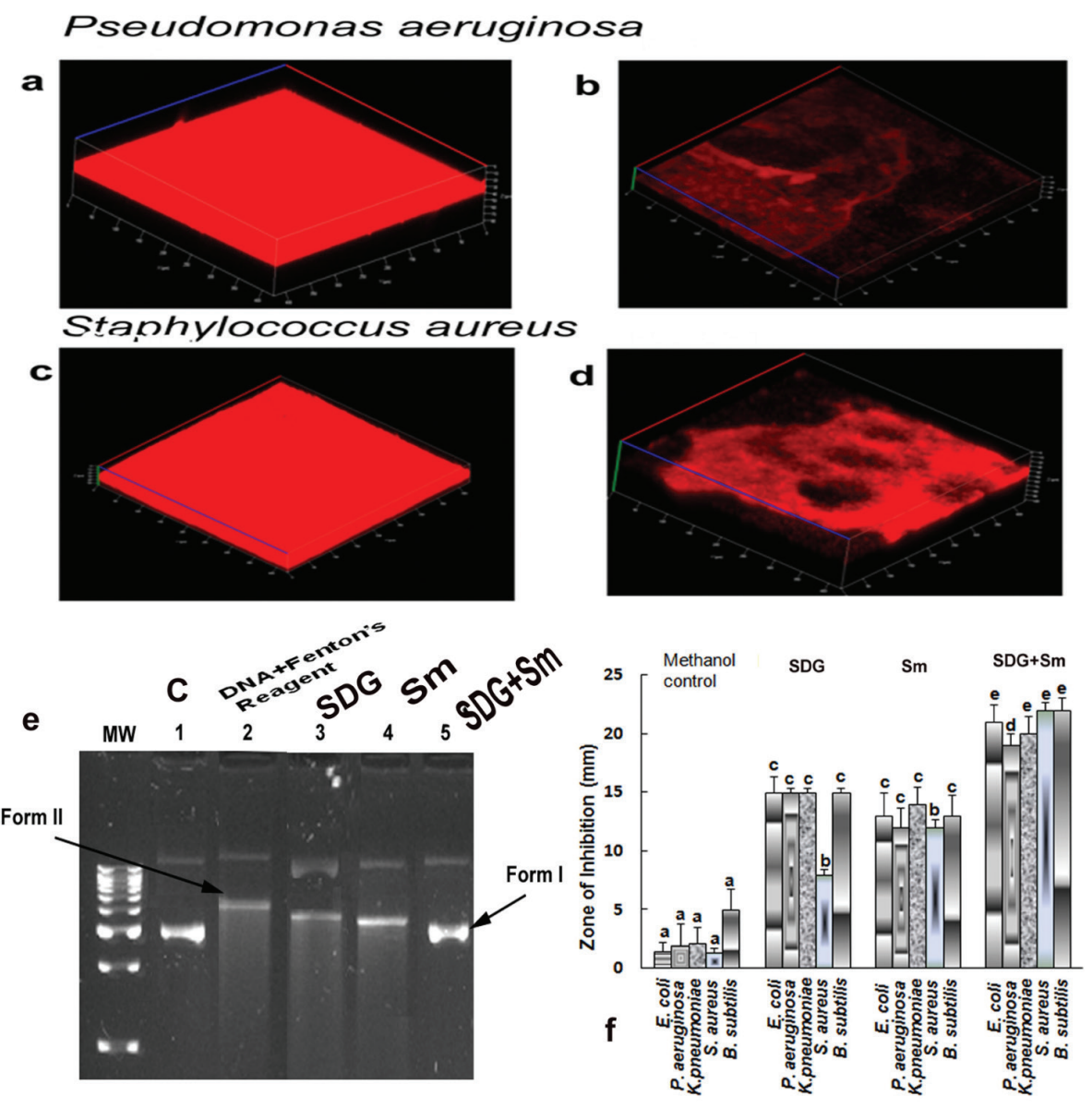

Figure 2. Biofilm inhibition analysis of SDG + Sm as analyzed by Confocal laser scanning microscopy. Control samples of tested bacteria, Staphylococcus aureus (a), Pseudomonas aeruginosa (c) formed an uninterrupted biofilm on the support. A significant biofilm inhibition as visualized by CLSM studies with break-down of the biofilm architecture and with decreased microcolonies on the cover-slips containing S. aureus (b), P. aeruginosa (d). (e) DNA scission assay: Effect of SDG, Sm, and SDG + Sm on the protection of DNA strand scission induced by hydroxyl radicals produced by Fenton reagent. M, DNA ladder (1 kb); Lane 1, pBR322 (native plasmid DNA); Lane 2, pBR322 DNA +Fenton reagent; Lane 3, pBR322 + SDG+ Fenton reagent; Lane 4: pBR322 + Sesamin + Fenton reagent; Lane 5, pBR322 + (SDG+Sm) + Fenton reagent. (2f) Quantitative results of antibacterial activity assayed by the disc diffusion method.

Table 3. Inhibition of 15-lipoxygenase and cyclooxygenase-2 assayed by human COX-2 inhibitor screening kit (Cayman, Ann Arbor, MI) by SDG, sesamin, and $\mathrm{SDG}+\mathrm{Sm}$.

\begin{tabular}{|c|c|c|c|}
\hline Samples & $\begin{array}{c}\text { Lipoxygenase } \\
\text { inhibition in \% } \\
\left(100 \mu \mathrm{g} \cdot \mathrm{ml}^{-1} \text { of }\right. \\
\text { samples })\end{array}$ & $\mathbf{I C}_{50}(\mu \mathrm{g})$ & $\begin{array}{c}\text { COX-2 inhibition } \\
\text { in \% (100 } \mu \text { g of } \\
\text { samples) }\end{array}$ \\
\hline SDG & 68.26 & $56 \pm 0.02$ & $62.18 \pm 3.14$ \\
\hline Sm & 67.07 & $58 \pm 0.23$ & $56 \pm 2.11$ \\
\hline $\mathrm{SDG}+\mathrm{Sm}$ & 94.37 & $35 \pm 053$ & $73.56 \pm 2.02$ \\
\hline
\end{tabular}

presented to a wellspring of hydroxyl radical, Fenton reaction. The parameters were 30 minutes at $37^{\circ} \mathrm{C}$. The plasmid DNA was damaged by Fenton reagent. It resulted in single-stranded (as indicated by arrows), cut DNA (Form II) supershift from native double-stranded DNA band (Form I) affirmed in agarose gel electrophoresis (Fig. 2e). In the examination, when plasmid DNA was pre-brooded for 30 minutes with SDG $(10 \mu \mathrm{g}), \mathrm{Sm}$ and their blend, SDG $+\mathrm{Sm}$ and presented to Fenton response under comparable conditions as above, SDG and Sm samples alone could prevent scission. SDG+Sm in combination offered prominent protection to DNA.

\section{CONCLUSION}

Value-added products could be ready to eat items. Due to their increased consumption globally large scale production is often the norm. A longer distribution period and greater distribution in longer distant regions have increased the complexities and relevance of food safety. The buyer many a time does not subject them to any further steps of processing prior to consumption. Further processing (washing or heating) could ensure effective nullification of any contaminants, including chemical residues or pathogenic bacteria. As a consequence, an increase in ready to eat produce related disease outbreaks has been reported. Pathogenic 
E. coli or Salmonella have been reported to survive on nitrile and latex gloves during and after the production of food items. The contamination complexity could be due to complex processes during manufacturing. In the majority of the cases, the use of contaminated water and manipulation by handlers without proper safety measures in place could be the reason for the transmission of bacterial contamination.Consumer awareness has prompted a particular interest in investigating natural antimicrobials as a preference. Spices are known for their distinctive aroma. They provide flavor to the food. They are classified as generally recognized as safe. These entities are rich sources of polyphenols. They are reported to exhibit their antimicrobial activity by forming complexes with soluble and extracellular protein entities.A class of secondary plant metabolites is lignans (iridoids). Two phenylpropanoid units dimerize oxidatively to produce them. Their molecular backbone consists of two $\mathrm{C} 6-\mathrm{C} 3$ phenylpropane units. Although comprising a simple backbone, lignans show an enormous structural diversity. A growing interest in these molecules arises due to their effectiveness in anticancer therapy. Aristolochia taliscana roots find use in traditional Mexican medicine contains neolignans. The Licarin A was found to be the most effective antimicrobial component. Licarin A was active against most variant and clinically isolated of Mycobacterium tuberculosis strains with MICs ranging from 3.12 to $12.5 \mathrm{mg} / \mathrm{ml}$. These studies provided an impetus for the present study, wherein, use of polyphenols in combination as an effective anti-microbial strategy could be employed [13].

An extraordinarily high concentration of lignans is reported in FS. It is particularly rich in the lignan SDG. It is also reported to contain small amounts of the matairesinol, pinoresinol, and isolariciresinol lignans.

Earlier studies have reported the use of synthetic SDG with a broad spectrum of antibacterial activity [14]. Sm, one of the major lignan of sesame oil has been reported with anti-bacterial capacity [15]. Anti-microbials could be as unrefined preparations (essential oils, extracts, and partially purified extracts) containing a wide assortment of mixes or might be an authentic molecule with an vigorous and exact activity.With this foundation set up, in the current examination, SDG and Sm were concentrated exclusively and also in combination. And the above combination examined, the SDG+Sm showed promising results. The HPLC fingerprint identified the presence of SDG and sesamin in seeds of FS and SS. SDG and sesamin were estimated to be 23.9 and 4.7 g. $\mathrm{kg}^{-1}$ of FS and SS, respectively. Disc diffusion-antimicrobial activity against E. coli, P. aeruginosa, K. pneumoniae, S. aureus, and B. subtilis the combination of SDG+Sm significantly arrests bacterial growth in comparison to independent use of SDG and Sm. Bacterial biofilm inhibition capacity of SDG+Sm $(750 \mathrm{mg})$ imaged by Confocal laser scanning microscopy revealed the loss of microcolonies. SDG+Sm when tested for 15-LOX inhibition, exhibited lower $\mathrm{IC}_{50}$ values (60\%) of $35 \pm 0.53$ when compared to $\mathrm{IC}_{50}$ values for SDG (56 \pm 0.02$)$ and $\mathrm{Sm}(58 \pm 0.23)$ by enzyme kinetics studies using spectrophotometric techniques. Similarly, SDG+Sm could inhibit the COX-2 enzyme at relatively lower concentrations. Furthermore, SDG+Sm quenched free radicals produced by Fenton's reagent studied by DNA-nicking assay indicating its strong antioxidant property with the capacity to protect DNA. Fortification of ready to eat food products at minute amounts of
SDG and sesamin could be attempted. The results of the present study encourage the use of FS and SS lignans in combination at $\mathrm{SDG}+\mathrm{Sm}$ in a wide range of applications as natural preservatives because it is also socio-culturally accepted food commodities. It could provide enough protection with pharmacological effects such as anti-inflammatory agents and aid in their promotion as nutraceuticals.

\section{ACKNOWLEDGMENT}

This work was financially supported by the University Grants Commission, New Delhi, India, and IOE - the University of Mysore sponsored by the MHRD (Ministry of Human Resource Development), Government of India, New Delhi, India for the facilities.

\section{CONFLICT OF INTEREST}

Authors declared that they do not have any conflicts of interest.

\section{FINANCIAL SUPPORT}

None.

\section{REFERENCES}

1. Gromadzka J, Wardencki W. Trends in edible vegetable oils analysis. Part A. Determination of different components of edible oils - a review. Pol J Food Nut Sci 2011;61:33-43; doi:10.2478/v10222-0110002

2. Parke DV, Lewis DFV. Safety aspects of food preservatives. Food Addi Contam 1992;9:561-77; doi:10.1080/02652039209374110

3. Davidson PM, Taylor MT. Chemical preservatives and natural antimicrobial compounds. In: Doyle $\mathrm{M}$ and Beuchat L (ed.). Food microbiology: fundamentals and frontiers, ASM, Washington DC, pp 713-45, 2007.

4. Mahboubi M, Kazempour N, Mahboubi A. The efficacy of essential oil as natural preservatives in vegetable oil. J Diet Suppl 2014;11:334 46; doi:10.3109/19390211.2014.887603

5. AOAC Association of Official Analytical Chemists. Official Method of Analysis of AOAC Intl. 16th edition. Method 991.43, St. Louis, MO, 1995.

6. Rajesha J, Kotamballi N, Chidambara M, Karun KM, Basavaraj M, Gokare AR. Antioxidant potentials of flaxseed by in vivo Model. J Agric Food Chem 2006;54:3794-9; doi:10.1021/jf053048a

7. Amarowicz R, Shahidi F, Pegg RB. Application of semipreparative RP-18 HPLC for the purification of sesamin and sesamolin. J Food Lipids 2001;8:85-94; doi:10.1111/j.1745-4522.2001.tb00186.x

8. Cai YQ, Luo Q, Sun M, Corke H. Antioxidant activity and phenolic compounds of 112 Chinese medicinal plants associated with anticancer. Life Sci 2004;74:2157-84; doi:10.1016/j.1fs.2003.09.047

9. Klancnik A, Piskernik BJ, Mozina SS. Evaluation of diffusion and dilution methods to determine the antibacterial activity of plant extracts. J Microbiol Methods 2010;81:121-6; doi:10.1016/j. mimet.20101.02.004

10. Zhang WP, Wang Y, Tian RM, Bougouffa S, Yang B, Cao HL, et al. Species sorting biofilm assembly by artificial substrates deployed in a cold seep system. Sci Rep 2014;4:1-7; doi.org/10.1038/srep06647

11. Rackova LM, Oblozinsky D, Kostalova VK, Bezakova L. Free radical scavenging activity and lipoxygenase inhibition of Mahonia aquifolium extract isoquinoline alkaloids. J Inflammation 2007;4:1521; doi:10.1186/1476-9255-4-15

12. Lee JC, Kim HR, Kim J, Jang YS. Antioxidant activity of ethanol extract of the stem of Opuntia ficus-indica var. saboten. J Agric Food Chem 2002;50:6490-6; https://doi.org/10.1021/jf020388c 
13. Trigo JP, Alexandra EMC, Saraiva JA, Pintado ME. High value added compounds from fruit and vegetable by products - characterization, bioactives and application in development of novel food products. Crit Rev Food Sci Nutr 2019;1-29; https://doi.org/10.1080/10408398.201 9.1572588

14. Mareai S, Khanum S, Rajesh J. Synthesis and evaluation of in vitro antibacterial properties of Secoisolariciresinol diglucoside. Int J Biochem Res Rev 2016;9:1-10; https://doi.org/10.9734/ IJBCR/2016/22277

15. Kumar CM, Singh SA. Bioactive lignans from sesame (S. indicum $L$.): evaluation of their antioxidant and antibacterial effects for food applications. J Food Sci Technol 2015;52:2934-41; https://doi. org/10.1007/s13197-014-1334-6
How to cite this article:

Bhaskar A, Sekhar S, Javaraiah R. Antibiofilm and antiinflammatory potential of lignans with collegial effect: Secoisolariciresinol diglucoside and sesamin as antimicrobial sources. J Appl Biol Biotech 2020;8(04):045-051. DOI: 10.7324/JABB.2020.80407 